Proceedings of the 46th International School and Conference on the Physics of Semiconductors "Jaszowiec" 2017, Szczyrk

\title{
Modeling of Transient Photocurrent in Organic Semiconductors Incorporating the Annihilation of Excitons on Charge Carriers
}

\author{
D. GŁowienkA* And J. SzMYTKOWski \\ Faculty of Applied Physics and Mathematics, Gdańsk University of Technology \\ G. Narutowicza 11/12, 80-233 Gdańsk, Poland
}

\begin{abstract}
The role of the annihilation of excitons on charge carriers has been theoretically investigated in organic semiconductors. We have developed the numerical drift-diffusion model by incorporation terms which describe the annihilation process. The transient photocurrent has been calculated for different injection barrier heights, exciton mobilities, and annihilation rate constants. We have demonstrated that the annihilation has a great influence on the range and the rising time of the photocurrent.
\end{abstract}

DOI: 10.12693/APhysPolA.132.397

PACS/topics: 71.35.-y, 73.50.-h, 73.50.Pz

\section{Introduction}

Organic semiconductors are materials that possess a few features which make them suitable to construct electronic devices, like solar cells, light-emitting diodes, and transistors. Most importantly, their cost of production is relatively low. In illuminated organic semiconductors, a photocurrent is a flow of charge carriers which are generated in the dissociation of excitons. However, a diffusion length of the excitons is much shorter than a thickness of material. Therefore any process, which influences a concentration of excitons, is relevant for final photocurrent and efficiency of the device $[1,2]$. A study of transient photocurrent allows to understand the dynamics of charge carriers generation mechanisms.

In this paper, we investigate the influence of excitons annihilation with charge carriers on transient photocurrent in organic semiconductors. In the simple explanation of this mechanism, an electron (hole) from an exciton interacts with a free hole (electron). It causes that the interacting charges annihilate and one hole (electron) is left from the exciton. Therefore, the concentration of free charge carriers does not change but the excitons concentration decreases. Considering a quantum mechanical description of this process, an energy of the quenched exciton is fully transferred to the interacting carrier via the Förster resonance energy transfer and, as a consequence, the exciton is lost. Recently, it has been proved that the effect of excitons annihilation visibly influences on photophysical and photoelectric processes in systems used in organic photovoltaics [3-6]. This fact is associated with a high probability of the annihilation which is described by high values of the annihilation rate constants $\left(10^{-15}-5 \times 10^{-14} \mathrm{~m}^{3} \mathrm{~s}^{-1}\right)[3,6]$.

*corresponding author; e-mail: dglowienka@mif.pg.gda.pl

\section{Model}

In order to model the photocurrent, the drift-diffusion set of equations has been considered [7, 8]. It should be mentioned that for the first time we incorporate the annihilation process in transient drift-diffusion model. To determine the electron $(n)$, hole $(p)$ and exciton $(X)$ concentrations, the following equations are used:

$$
\begin{aligned}
& \frac{\partial^{2} \phi}{\partial x^{2}}=-\frac{q}{\varepsilon_{0} \varepsilon_{r}}(p-n), \\
& \frac{\partial n}{\partial t}=k_{\text {diss }}(E) X-R_{L}(n, p)+\frac{1}{q} \frac{\partial}{\partial x} J_{n}, \\
& \frac{\partial p}{\partial t}=k_{\text {diss }}(E) X-R_{L}(n, p)-\frac{1}{q} \frac{\partial}{\partial x} J_{p}, \\
& \frac{\partial X}{\partial t}=G+\frac{1}{4} R_{L}(n, p)-\frac{X}{\tau_{X}}-k_{\text {diss }}(E) X-\gamma_{n X} n X \\
& \quad-\gamma_{p X} p X-\frac{1}{q} \frac{\partial}{\partial x}\left(-\mu_{X} k_{\mathrm{B}} T \frac{\partial X}{\partial x}\right) .
\end{aligned}
$$

Equation (1) is the Poisson equation used to calculate the local electric potential $\phi$ and the electric field $E$ in space $(x)$ and time $(t)$. The parameter $q$ is the elementary charge, $\varepsilon_{0}$ denotes the vacuum permittivity and $\varepsilon_{r}$ is the permittivity of the material. Equations (2)(4) represent the time dependent continuous equations which implement gradients of concentration fluxes (density currents) of charge carriers or excitons. The symbol $k_{\mathrm{B}}$ denotes the Boltzmann constant, $T$ represents the absolute temperature and $\mu_{X}$ is the excitons mobility. Other terms in the continuous equations describe the generation (with a plus sign) or the decay (with a minus sign) processes for $n, p$ and $X$. The parameter $G$ is excitons generation rate, $\tau_{X}$ represents the lifetime of excitons, and $\gamma_{p X}$ and $\gamma_{n X}$ are the rate constants, which are associated with the excitons annihilation with holes and electrons, respectively. The electric field dependent rate $k_{\text {diss }}$ describes a dissociation of excitons into separated electrons and holes, which is explained by 
the Onsager-Braun model [9]. The bimolecular recombination term can be given by $R_{L}(n, p)=\gamma_{L}\left(n p-n_{\text {int }}^{2}\right)$, where $\gamma_{L}=q\left(\mu_{n}+\mu_{p}\right) / \varepsilon_{0} \varepsilon_{r}$ is a Langevin recombination coefficient and $n_{\text {int }}$ represents the intrinsic concentration of charge carriers in the material. The parameters $\mu_{n}$ and $\mu_{p}$ are the mobilities of electrons and holes, respectively. The factor $1 / 4$ in Eq. (4) is due to creation of excitons from bimolecular recombination of charge carriers with $25 \%$ probability.

The total photocurrent is a sum of electrons $\left(J_{n}\right)$, holes $\left(J_{p}\right)$ and displacement $\left(J_{\text {disp }}\right)$ currents

$$
\begin{aligned}
& J_{n}=-q n \mu_{n} \frac{\partial \phi}{\partial x}+\mu_{n} k_{\mathrm{B}} T \frac{\partial n}{\partial x} \\
& J_{p}=-q p \mu_{p} \frac{\partial \phi}{\partial x}-\mu_{p} k_{\mathrm{B}} T \frac{\partial p}{\partial x}
\end{aligned}
$$

and

$$
J_{\text {disp }}=\varepsilon_{r} \varepsilon_{0} \frac{\partial E}{\partial t} .
$$

In order to solve the equations numerically, they were discretized with the Sharfetter-Gummel method [10]. To define the dynamics of photocurrent (a rise time), an establishment of equilibrium state is used. Here, the steady-state condition has been chosen as $\left|\left(J_{\text {total }}^{t+1}-J_{\text {total }}^{t}\right) / J_{\text {total }}^{t}\right| \leq 10^{-4}$. In the paper, the shortcircuit photocurrent is calculated.

The lifetime of excitons $\tau_{X}$ is assumed as $1 \mu \mathrm{s}$. The initial separation distance between charge carriers is equal to $1.3 \mathrm{~nm}$. The device thickness $L=120 \mathrm{~nm}$, the temperature $T=293 \mathrm{~K}$ and $\varepsilon_{r}=3.4$. The values of built-in voltage $V_{\text {built }}$ across the device and energy band-gap $E_{g}$ are equal to $1.34 \mathrm{~V}$ and $0.8 \mathrm{eV}$, respectively.

For boundary conditions, we assume $n(0)=$ $N_{c} \exp \left(-\phi_{n} / k_{\mathrm{B}} T\right)$ and $n(L)=N_{c} \exp \left(\frac{\phi_{p}-E_{g}}{k_{\mathrm{B}} T}\right)$ for electrons. In the case of holes, the boundary concentrations are given by $p(0)=N_{v} \exp \left(\frac{\phi_{n}-E_{g}}{k_{\mathrm{B}} T}\right)$ and $p(L)=$ $N_{v} \exp \left(-\phi_{p} / k_{\mathrm{B}} T\right)$. The effective density of states in conduction $\left(N_{c}\right)$ and valence $\left(N_{v}\right)$ bands are equal to $2.5 \times 10^{25} \mathrm{~m}^{-3}$. For simplicity, we consider the same values of the Schottky barrier heights for electrons $\left(\phi_{n}\right)$ and holes $\left(\phi_{p}\right)$. Also, the same mobilities $\left(10^{-7} \mathrm{~m}^{2} \mathrm{~V}^{-1} \mathrm{~s}^{-1}\right)$ and equal magnitudes of annihilation rate constants are chosen for both types of charge carriers. It causes that the transient concentration profiles are similar for both electrons and holes. Thus, it is convenient to use notation $\gamma_{c X}$ and $\phi_{c}$, with $c \in\{n, p\}$, in further discussion. Depending on the injection barrier height, the Schottky $\left(\phi_{c} \neq 0\right)$ and the ohmic $\left(\phi_{c}=0\right)$ contacts are defined.

The model is solved forward in time with turn-on method [11]. First, the dark currents $(G=0)$ are simulated for $0.5 \mu \mathrm{s}$ and then sample is illuminated with $G=2.7 \times 10^{27} \mathrm{~m}^{-3} \mathrm{~s}^{-1}$ for $4 \mu \mathrm{s}$. Finally, the dark conditions are simulated up to $9 \mu \mathrm{s}$.

\section{Results and discussion}

The terms, which describe the annihilation process in Eq. (4), depend on three parameters: charge carriers con- centration, excitons concentration and annihilation rate constants. Therefore, we will analyze how these parameters influence on transient photocurrents. The results are presented in the middle of material, because the dynamics of photocurrent is independent of the position. In addition, we decided to illustrate obtained results using both absolute and normalized scales in all figures.

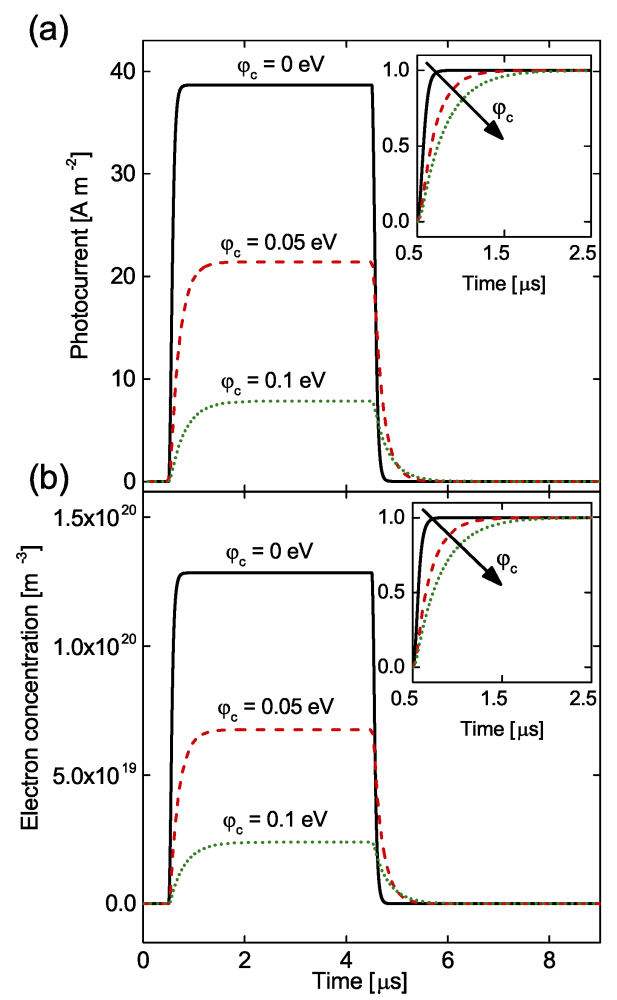

Fig. 1. Transient photocurrent (a) and concentration of electrons versus time (b) both calculated with different injection barrier heights in the absence of annihilation process. Excitons mobility is equal to $10^{-7} \mathrm{~m}^{2} \mathrm{~V}^{-1} \mathrm{~s}^{-1}$ for both cases. The vertical axes in insets show values of normalized photocurrent (a) and normalized concentration (b).

Figure 1a shows transient photocurrents calculated for different injection barrier heights. We can see that a lowering of injection barrier leads to an increase of photocurrent magnitude. The rise time is the shortest $(0.2 \mu \mathrm{s})$ for ohmic contact and increases for the Schottky contacts (up to $0.64 \mu \mathrm{s}$ for $0.1 \mathrm{eV}$ ). The explanation is that $\phi_{c}$ impacts directly on the concentration of charge carriers, as shown in Fig. 1b. The annihilation of excitons gives no visible influence on the dynamics of photocurrent for ohmic contacts due to short rise times (not shown). Thus, next simulations have been carried out only for the Schottky contacts.

Figure 2a demonstrates the impact of excitons mobility on transient photocurrents. We can see that the magnitude of photocurrent is lower for higher $\mu_{X}$. This result may be explained by the effect of charge carriers photogeneration due to the excitons dissociation. If the mo- 
(a)

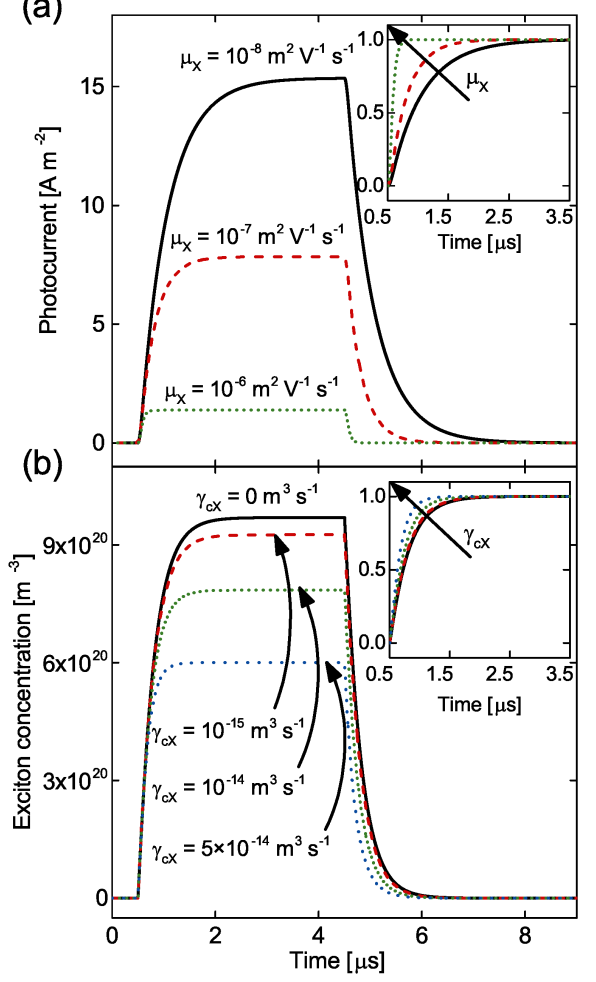

Fig. 2. Transient photocurrent without annihilation process for different mobilities of excitons (a) and concentration of excitons as a function of time for varied annihilation rates with $\mu_{X}=10^{-7} \mathrm{~m}^{2} \mathrm{~V}^{-1} \mathrm{~s}^{-1}$ (b). Results of simulation are plotted for injection barrier equal to $0.1 \mathrm{eV}$. The vertical axes in insets show values of normalized photocurrent (a) and normalized concentration (b).

bility is higher, the concentration of excitons decreases and, as a consequence, the photocurrent is getting lower. Apart from the photocurrent magnitude, the influence of exciton mobility on photocurrent's dynamics is visible (inset in Fig. 2a). When the mobility of excitons is higher, then the dynamics is much faster. The rise time is equal to $0.23 \mu \mathrm{s}$ for $\mu_{X}=10^{-6} \mathrm{~m}^{2} \mathrm{~V}^{-1} \mathrm{~s}^{-1}$ and increases up to $0.84 \mu \mathrm{s}$ for $\mu_{X}=10^{-8} \mathrm{~m}^{2} \mathrm{~V}^{-1} \mathrm{~s}^{-1}$. The concentration of excitons as a function of time is presented in Fig. $2 b$ for different values of annihilation rate constants. We can see that the annihilation process causes a decrease of the $X$ magnitude. The lowering concentration of excitons should lead to faster response of photocurrent, because less number of excitons dissociates into seperated charge carriers and the steady-state condition is achieved faster.

Figure 3 shows the influence of annihilation process on the dynamics of photocurrent calculated for different exciton mobilities. For each mobility, the annihilation rate is given for three values: low $\left(10^{-15} \mathrm{~m}^{3} \mathrm{~s}^{-1}\right)$, moderate $\left(10^{-14} \mathrm{~m}^{3} \mathrm{~s}^{-1}\right)$ and high $\left(5 \times 10^{-14} \mathrm{~m}^{3} \mathrm{~s}^{-1}\right)$. We can see that the impact of excitons annihilation is the most evident for the lowest mobility $\left(\mu_{X}=10^{-8} \mathrm{~m}^{2} \mathrm{~V}^{-1} \mathrm{~s}^{-1}\right)$. In

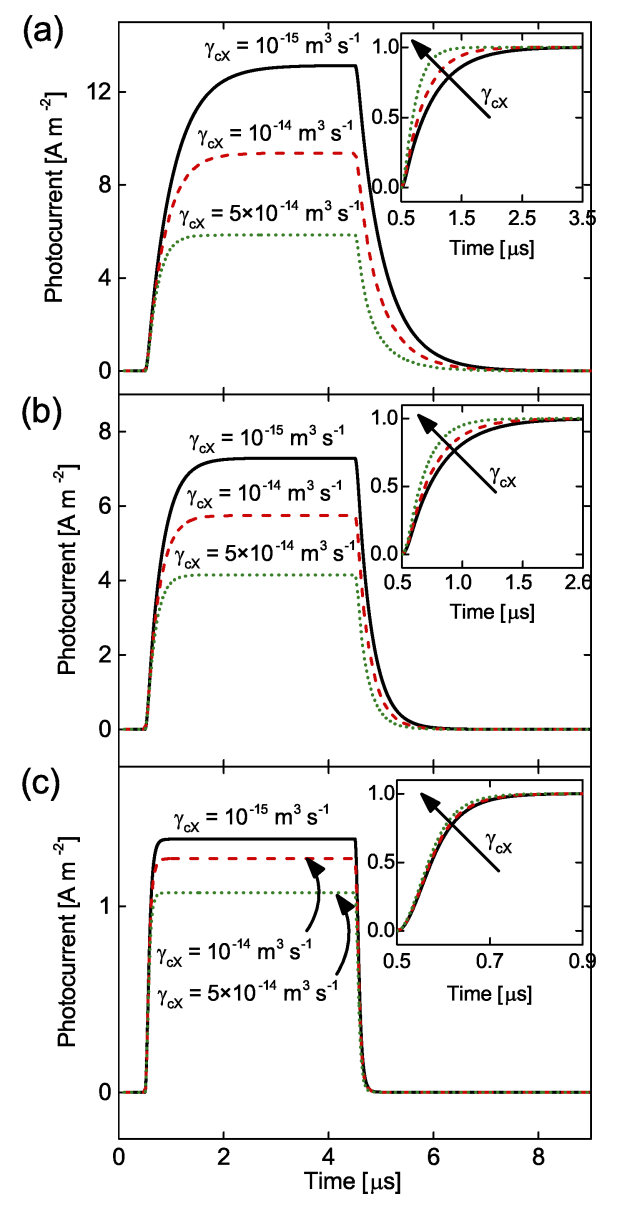

Fig. 3. Transient photocurrent for different mobilities of excitons calculated for three annihilation rate constants. Excitons mobility equals to $10^{-8} \mathrm{~m}^{2} \mathrm{~V}^{-1} \mathrm{~s}^{-1}$ (a), $10^{-7} \mathrm{~m}^{2} \mathrm{~V}^{-1} \mathrm{~s}^{-1}$ (b), and $10^{-6} \mathrm{~m}^{2} \mathrm{~V}^{-1} \mathrm{~s}^{-1}$ (c). The height of injection barrier for the Schottky contacts is equal to $0.1 \mathrm{eV}$. The vertical axes in insets show normalized photocurrent.

this case, the rising time is $0.83 \mu \mathrm{s}$ for the lowest value of $\gamma_{c X}$ and decreases to $0.53 \mu$ s when the probability of annihilation increases (inset in Fig. 3a). If the mobility of excitons is $\mu_{X}=10^{-7} \mathrm{~m}^{2} \mathrm{~V}^{-1} \mathrm{~s}^{-1}$, we observe lower response of the annihilation to photocurrent dynamics. Here, the rising time is equal to $0.62 \mu \mathrm{s}$ for very low annihilation rate constant, and reaches a value of $0.45 \mu \mathrm{s}$ when $\gamma_{c X}$ increases (Fig. 3b). For the mobility of excitons equal to $10^{-6} \mathrm{~m}^{2} \mathrm{~V}^{-1} \mathrm{~s}^{-1}$, the variation of annihilation rate changes the rise time of photocurrent negligibly (inset in Fig. 3c).

\section{Conclusions}

We have studied the influence of excitons annihilation with charge carriers on transient photocurrent generated in organic semiconductor. It has been found out that an increase of the injection barrier height lowers the dynamics of photocurrent which is directly associated with 
charge carrier concentration. In contrary, higher values of the exciton mobility and the annihilation rate constant lead to decrease of photocurrent rise time due to changes of excitons concentration. The obtained results demonstrate that the annihilation process has the highest impact for structures with lower exciton mobility and higher (the Schottky-type) injection barrier. It has been shown that an increase of the annihilation rate constant up to $5 \times 10^{-14} \mathrm{~m}^{3} \mathrm{~s}^{-1}$ (a similar value to an experimental result for P3HT [3], which is frequently used in organic photovoltaics) leads to lower magnitudes of photocurrent. This fact confirms that the exciton annihilation mechanism should play a significant role in structures applied in organic solar cells.

\section{References}

[1] P.W.M. Blom, V.D. Mihailetchi, L.J.A. Koster, D.E. Markov, Adv. Mater. 19, 1551 (2007).

[2] P. Peumans, A. Yakimov, S.R. Forrest, J. Appl. Phys. 104, 3693 (2003).
[3] A.J. Ferguson, N. Kopidakis, S.E. Shaheen, G. Rumbles, J. Phys. Chem. C 112, 9865 (2008).

[4] I.A. Howard, J.M. Hodgkiss, X. Zhang, K.R. Kirov, H.A. Bronstein, C.K. Williams, R.H. Friend, S. Westenhoff, N.C. Greenham, J. Am. Chem. Soc. 132, 328 (2010).

[5] J. Szmytkowski, Phys. Status Solidi RRL 6, 300 (2012).

[6] B. Verreet, A. Bhoolokam, A. Brigeman, R. Dhanker, D. Cheyns, P. Heremans, A. Stesmans, N.C. Giebink, B.P. Rand, Phys. Rev. B 90, 115304 (2014).

[7] I. Hwang, C.R. Mcneill, N.C. Greenham, J. Appl. Phys. 106, 094506 (2009).

[8] Z.S. Wang, W.E.I. Sha, W.C.H. Choy, J. Appl. Phys. 120, 213101 (2016).

[9] C.L. Braun, J. Chem. Phys. 80, 4157 (1984).

[10] D.L. Scharfetter, H.K. Gummel, IEEE Trans. Electron. Dev. 16, 64 (1969).

[11] I. Hwang, N.C. Greenham, Nanotechnology 19 , 424012 (2008). 